UCRL-ID-126179

\title{
Spheromak Physics Development
}

\author{
E. B. Hooper
}

January 27, 1997

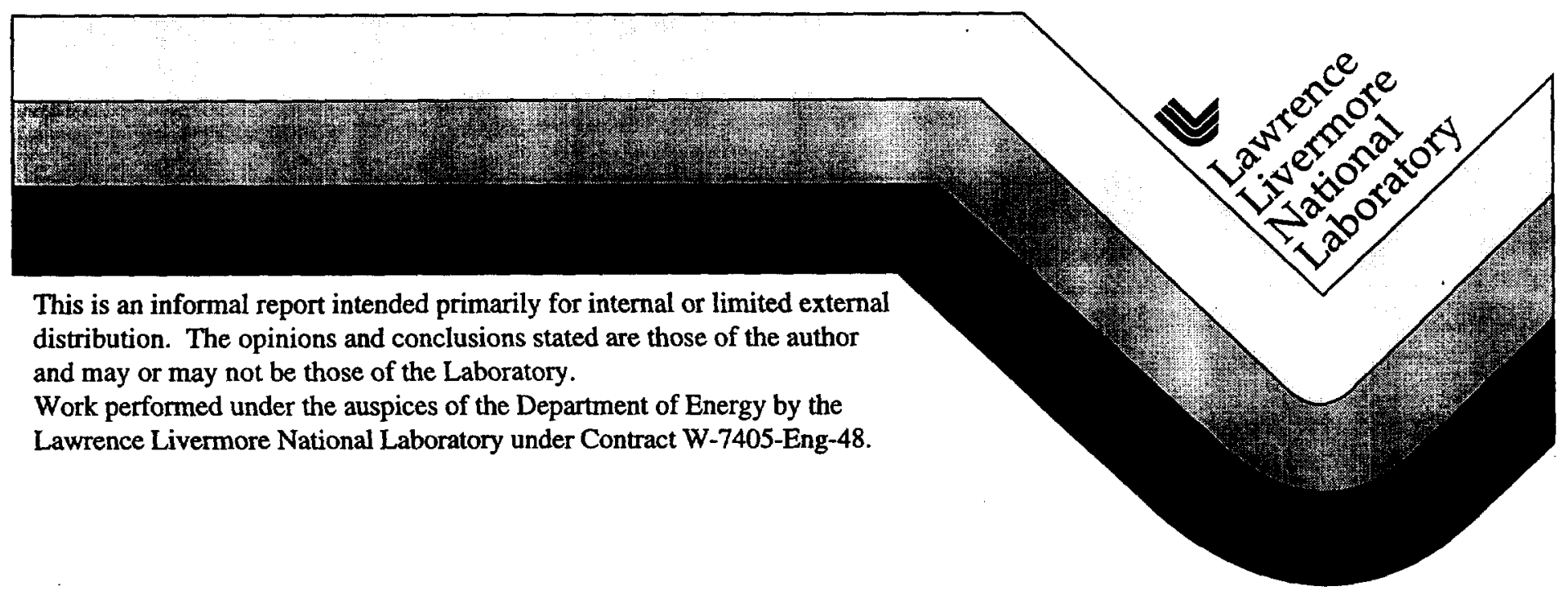




\section{DISCLAIMER}

This document was prepared as an account of work sponsored by an agency of the United States Government. Neither the United States Government nor the University of California nor any of their employees, makes any warranty, express or implied, or assumes any legal liability or responsibility for the accuracy, completeness, or usefulness of any information, apparatus, product, or process disclosed, or represents that its use would not infringe privately owned rights. Reference herein to any specific commercial product, process, or service by trade name, trademark, manufacturer, or otherwise, does not necessarily constitute or imply its endorsement, recommendation, or favoring by the United States Government or the University of California. The views and opinions of authors expressed herein do not necessarily state or reflect those of the United States Government or the University of California, and shall not be used for advertising or product endorsement purposes.

This report has been reproduced directly from the best available copy.

Available to DOE and DOE contractors from the Office of Scientific and Technical Information P.O. Box 62, Oak Ridge, TN 37831

Prices available from (615) 576-8401, FTS 626-8401

Available to the public from the

National Technical Information Service

U.S. Department of Commerce 5285 Port Royal Rd.

Springfield, VA 22161 
Spheromak Physics Development

\author{
E. B. Hooper
}

January 27, 1997 


\section{Summary}

The spheromak is a Magnetic Fusion Energy (MFE) configuration, which is a leading alternative to the tokamak. It has a simple geometry which offers an opportunity to achieve the promise of fusion energy if the physics of confinement, current drive, and pressure holding capability extrapolate favorably to a reactor.

Recent changes in the US MFE program, taken in response to budget constraints and programmatic directions from Congress, include a revitalization of an experimental alternative concept effort. Detailed studies of the spheromak were consequently undertaken to examine the major physics issues which need to be resolved to advance it as a fusion plasma, the optimum configuration for an advanced experiment, and its potential as a reactor.

As a result of this study, we conclude that it is important to evaluate several physics issues experimentally. Such an experiment might be appropriately be named the Sustained Spheromak Physics Experiment (SSPX). It would address several critical issues, the solution to which will provide the physics basis to enable an advanced experiment. The specific scientific goals of SSPX would be to:

- Demonstrate that electron and ion temperatures of a few hundred electron volts can be achieved in a steady-state spheromak plasma sustained by a magnetic dynamo ("helicity injection").

- Relate energy confinement quantitatively to the magnetic turbulence accompanying the dynamo and use this knowledge to optimize performance.

- Measure the magnetic field profiles and magnetic turbulence in the plasma and relate these to the science of the magnetic dynamo which drives the current in the plasma.

- Examine experimentally the pressure holding capability ("beta limit") of the spheromak.

- Understand the initial phases of the transition of the plasma from an equilibrium supported by a magnetic-flux conserving wall to one supported by external coils.

These goals could be achieved in an experiment with duration of a few milliseconds, and can consequently be addressed at a relatively low cost. There are additional goals which would be addressed in a larger, follow-up experiment, the Advanced Spheromak Physics Experiment. These include 
the achievement of temperatures in the multi-keV range, the control of low mode-number instabilities (perhaps with a feedback system), and the technology of long-pulse current drive.

This document reviews past work in the field and describes a number of new results. Recent publications which complement this report are also referenced. These publications also describe the characteristics of an experiment to examine the important spheromak physics issues. 


\section{Table of contents}

Summary

1.0 Why a new spheromak experiment?

5

1.1 Reasons and plans

5

1.2 Fundamental science

6

1.3 Opportunity for innovation

7

2.0 Background

2.1 Motivation

7

2.2 History

8

3.0 Reactor Opportunities

Acknowledgment

References 


\subsection{Why a new spheromak experiment?}

\subsection{Reasons and plans}

Previous research, both experimental and theoretical, is summarized in detail in the next section. Significant progress was made throughout the course of this research, but experiments were terminated before the lessons learned could be applied fully to magnetic fusion energy issues. Recent theory and review of data indicate a need for an experiment to evaluate the critical, fusion related issues, especially core energy confinement of the plasma and the beta ( $\beta$, ratio of kinetic to magnetic pressures) limit, with the goal of developing a small fusion reactor with low cost of electricity.

Previous experimental progress, especially in the CTX experiment at Los Alamos, resulted from progressive improvements in vacuum techniques, in controlling the open magnetic flux surrounding the toroidally confined plasma, and in the means for supplying magnetic helicity (current drive) to the spheromak. Each of these steps resulted from experiment and the interpretation of diagnostic measurements. An electron temperature of 400 $\mathrm{eV}$ and an ion temperature of $1 \mathrm{keV}$ were obtained in an experiment which had the goal of compressing a spheromak with high explosives accelerating a thin section of the flux conserver wall to high velocity. (The compression experiment was not carried out.) Because this experiment was not directed to magnetic fusion energy issues, electron temperature and energy confinement measurements were not focused on the steady-state (sustainment) phase of operation. Thus, although the high temperature results are very exciting, they cannot be used with confidence to project parameters in fusion experiments and reactors.

Projecting spheromak performance requires measurement of $T_{e}$ and energy confinement during sustainment, and to relate the energy losses to the level of magnetic turbulence which accompanies the magnetic dynamo which balances the ohmic losses of current. To succeed, any new experiment must apply modern vacuum techniques, largely learned from tokamak research which demonstrated their importance, to the experiment with the goal of maximizing temperatures.

- Titanium gettering was used to minimize impurities in spheromak plasmas. It was found, however, that the plasma pulse length was limited by heating of the flux-conserver surface, liberating large amounts of hydrogen. New vacuum techniques using low- $Z$ gettering have been developed on tokamaks in recent years and can overcome this problem. The experiment proposed here will be designed to utilize low- $Z$ gettering, especially boronization, in addition to bake-out and discharge cleaning, to control the impurities and fuel gas $\left(\mathrm{H}_{2}\right.$ or $\left.\mathrm{D}_{2}\right)$. 
- Energy confinement was found to be sensitive to the amount of magnetic flux surrounding the plasma which was open to nearby walls. Current flowing on the resistive, cold plasma on this flux was a large energy loss mechanism in most experiments. Careful control of magnetic errors and open flux was essential for the high-temperature experiment. Further control of the flux coupling between the gun and the spheromak during the sustainment phase is planned and should further improve the global confinement.

More recently, Fowler ${ }^{1}$ hypothesized that the core energy confinement is much better than the global confinement measured in the experiments, and an extensive review by Hooper, et al. $^{2}$ of the data in decaying spheromak plasmas is consistent with this hypothesis. Fowler also applied concepts of resistive tearing mode turbulence to the problem of energy confinement, concluding that a reactor-grade spheromak should have good confinement if the hypothesis is true. The experimental test of core-confinement in a sustained plasma is a major goal of the proposed experiments; they will be accompanied by measurements of the magnetic turbulence associated with the magnetic dynamo. Following experiments on energy confinement during sustainment, tests can be made of improvements on the pressure stability limit, beta limits, and their relationship to confinement.

\subsection{Fundamental science}

The fundamental science behind the spheromak experiment is that of the magnetic dynamo, reconnection of magnetic fieldlines, and resultant current drive. The dynamo physics is a laboratory version of the mechanism which generates the magnetic field of the earth and sun, and which plays a major role in many astrophysical phenomena. We know from Cowling's theorem ${ }^{3}$ that the generation of an axisymmetric magnetic field from the dynamo requires a breaking of axisymmetry in the plasma flow and magnetic fields which constitute the dynamo. The spheromak (and closely related reversedfield pinch, RFP) operate at high magnetic Reynolds number ("Lundquist number"), with the result that reconnection of the magnetic field to generate the confining, axisymmetric field, must occur over distances much shorter than the size of the device. The primary mechanism for this to happen is the generation of magnetic turbulence through the nonlinear interaction of tearing modes in the plasma, although distortion of the axisymmetric configuration by global modes can generate a dynamo in the edge boundary layer of the plasma through time-varying stochastic fields.

Magnetic turbulence is less well understood than ordinary fluid turbulence or even electrostatic turbulence in tokamak plasmas. The experimental challenge is to measure the characteristics of this turbulence sufficiently well that the data contributes to the fundamental understanding 
of the turbulence, and to minimize its effects on the energy losses from the magnetic configuration thereby moving the spheromak further into the fusion regime. The measurements cannot be made by local magnetic probes without destroying the high temperature characteristic of the plasma being studied, so new diagnostic techniques will need to be applied.

The experimental study in turn will challenge the development of theory and computational modeling of the magnetic processes. We anticipate that the results (with those from RFPs) will provide a stimulus for a better understanding of the dynamo and reconnection processes throughout the universe.

\subsection{Opportunity for innovation}

Although the immediate goals of the experiment are the evaluation of energy confinement and other important physics of the spheromak, it will also provide an opportunity for innovative concepts to be explored and applied to the spheromak. To enhance the possibility for this to occur, collaborations both within LLNL and throughout the community will be encouraged. One purpose of these collaborations will be to draw upon the ideas from a wide range of physicists.

\subsection{Background}

\subsection{Motivation}

The CTX experiment at Los Alamos obtained ${ }^{4} T_{e} \approx 400 \mathrm{eV}$ at densities of several $\times 10^{20} \mathrm{~m}^{-3}$. There is a great similarity between this accomplishment and the early, important success in tokamaks, namely the T3 results ${ }^{5}$ reported at the IAEA meeting at Novosibirsk in 1968. At the time it was reported that T3 attained $T_{i} \approx 400 \mathrm{eV}, n \sim 5 \times 10^{20} \mathrm{~m}^{-3}$, and an estimated $T_{e}$ in the range $0.1-2$ $\mathrm{keV}$ with a $\tau_{E}$ up to $10 \mathrm{msec}$. It was subsequently shown, with Thomson scattering by the English team, that $T_{e} \sim 400 \mathrm{eV}$; this was reported at the Dubna meeting in 1969. It is generally believed that the most important achievement was the electron temperature which demonstrated that electrons were well confined, and it was considered that T3 constituted the "proof-of-principle" for tokamaks.

The solid flux conserver experiments in CTX referenced above had a similar electron temperature (indicating toroidal confinement of electrons), had similar ion temperatures, and had densities in the mid $10^{20} \mathrm{~m}^{-3}$ range. The CTX results show that spheromaks can confine electrons at interesting temperatures, although the global energy confinement was poorer, of order $100 \mu \mathrm{sec}$. Fowler conjectured ${ }^{6,7,8}$ that the core energy confinement was much 
better than the global energy confinement (perhaps by as much as a factor of 10 ), and if this can be achieved in a sustained configuration the result would, in a way similar to T3, be a "proof-of-principle" for spheromaks.

A spheromak reactor ${ }^{9}$ is projected to be much smaller than a tokamak, lacks toroidal field coils, and is generally much simpler. We estimate that, if the physics can be demonstrated, the cost of electricity for a $1 \mathrm{GW}$ plant will be comparable to that from a fission plant. The potential payoff of research on the spheromak is consequently very large.

\subsection{History}

Interest in the spheromak was generated in the late 70s with a groundbreaking paper by Rosenbluth and Bussac ${ }^{10}$ concerning MHD equilibrium and stability of the device. The spheromak is a toroidal magnetic configuration with helical field lines lying on closed surfaces as in the tokamak. Figure 1 shows the basic geometry as initially modeled; we will later consider changes to this which have evolved as current drive techniques were developed.

The spheromak requires no toroidal field coils, thus offering the possibility of a greatly simplified, compact reactor. Large currents flowing within the plasma produce the toroidal and poloidal fields, which are of comparable magnitude. The radial equilibrium force is held by an external vertical field or (for short time scales) fields produced by eddy currents in a tight-fitting conducting shell.

Five different methods for forming the spheromak have been proposed and tested: an inductive flux-core scheme, ${ }^{11}$ a theta $/ z$-pinch, ${ }^{12}$ a magnetized coaxial gun, ${ }^{13},{ }^{14}$ a conical z-pinch, ${ }^{15}$ and a kinked z-pinch. ${ }^{16}$ Experiments based on each of these concepts successfully demonstrated spheromak formation, although the best performance in confinement and electron temperature were eventually achieved in gun-produced spheromaks: electron temperature $=400 \mathrm{eV}$, peak magnetic field $=3 \mathrm{~T}$, toroidal magnetic current $=$ $1 \mathrm{MA}$, helicity decay time of $2 \mathrm{~ms}$, and global energy decay time of $0.2 \mathrm{~ms}^{4}$

The magnetized coaxial plasma gun technique was pioneered at LLNL and LANL in the late 70s and early 80s with the BETA-II and CTX

experiments. Plasma flowing from the coaxial gun discharge entrains poloidal magnetic field generated by solenoidal coils. (C.f. Fig. 2) The plasma and fields flow into a formation region where the spheromak is established following magnetic reconnection. Early work showed that spheromaks could readily be formed by this technique, although the discharges were radiation dominated from large quantities of low- $Z$ impurities, with electron temperatures in the10 $\mathrm{eV}$ range. ${ }^{17}$ It was also demonstrated that a close fitting, oblate, fluxconserving shell was needed to suppress tilt and shift instabilities. ${ }^{18,19,20}$ The ability of the coaxial gun to form the spheromak within a flux conserver ${ }^{21}$ is a 


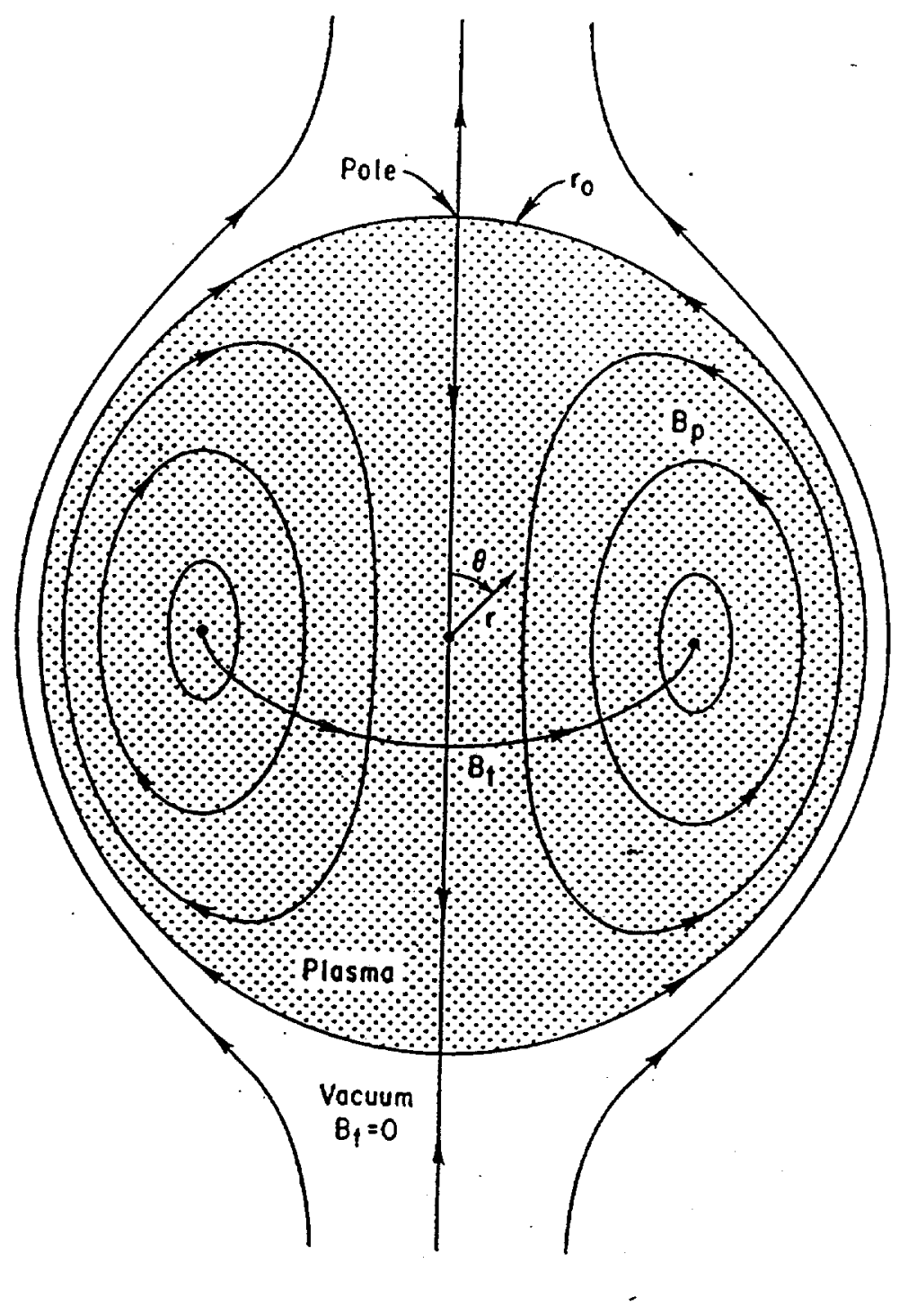

Fig. 1. The original spheromak geometry of Rosenbluth and Bussac. Note that the magnetic $x$-points lie on the geometric axis, so that there is no plasma between the separatrix and this axis. 
major advantage of this technique and may account for why it proved the most successful.

Spheromak research made a large amount of progress in a little more than a decade, in part because of the existence of MHD theory and the Taylor minimum energy principle ${ }^{22}, 23$ which provided a sound theoretical basis for the work. Indeed, a key result of the early spheromak experiments at LLNL and elsewhere was the validation of the Taylor theory. Taylor applied relaxation concepts to reversed field pinch discharges, concepts originally put forward by L. Woltjer ${ }^{24}$ for astrophysical plasmas and later by Wells ${ }^{25}$ to explain early compact toroid results. The spheromak can be viewed as the low-aspect-ratio, zero-reversal limit of the RFP, not requiring external toroidal field. Spheromaks exhibited "flux amplification" and other phenomena that could not be explained by the usual models, ${ }^{17}$ but were in quantitative agreement with Taylor's theory. The singular importance of the magnetic helicity ${ }^{22,26}$ was demonstrated, ${ }^{27}$ which led to the concept of helicity injection for the long-pulse build up and sustainment of the spheromak over many resistive decay times. ${ }^{28}$

Spheromak confinement experiments at LLNL concluded in 1982, although experiments on related compact tori continued for other purposes. Gun-produced spheromak experiments at Osaka ${ }^{14,29}$ and at Nihon ${ }^{30}$ Universities in Japan began in about 1980. At LANL, the CTX experiment continued confinement studies that led to improved plasma parameters. LANL developed the slow (100-1000 microsecond) formation technique, ${ }^{28}$ which was subsequently employed on compact tori at LLNL to produce cleaner discharges. Through a sequence of improvements in plasma-facing surfaces, flux-conserver designs to minimize losses on open field lines at the plasma edge, discharge cleaning, and titanium gettering, the plasma purity and lifetime on CTX were improved. Flattops of almost $6 \mathrm{~ms}^{31}$ and total duration of nearly $10 \mathrm{~ms}^{28}$ were achieved by halting the ohmic decay of the spheromak by continuous helicity injection from the gun. Experiments on the large flux conserver reached $200 \mathrm{eV}$ electron temperatures. ${ }^{32}$ The parameters were limited by the onset of an interchange instability when the central beta reached $\sim 20 \%$, but the global beta was only a few percent. Theoretical calculations $s^{33,34,35}$ indicate that correct shaping of the flux conserver can increase the magnetic shear of the spheromak equilibrium, leading to global $\beta$-limits of $10 \%$ or more. (Shaping is also required to stabilize the tilt and shift modes. ${ }^{10,36}$ ) In a smaller flux conserver, a field of $3 \mathrm{~T}$, electron density of $5 \times 10^{20} \mathrm{~m}^{-3}$, electron temperature of $400 \mathrm{eV}$, ion temperature of $1 \mathrm{keV}$, and a global confinement parameter $n \tau=1.8 \times 10^{16} \mathrm{~m}^{-3}$ sec were reached. ${ }^{37}$ 

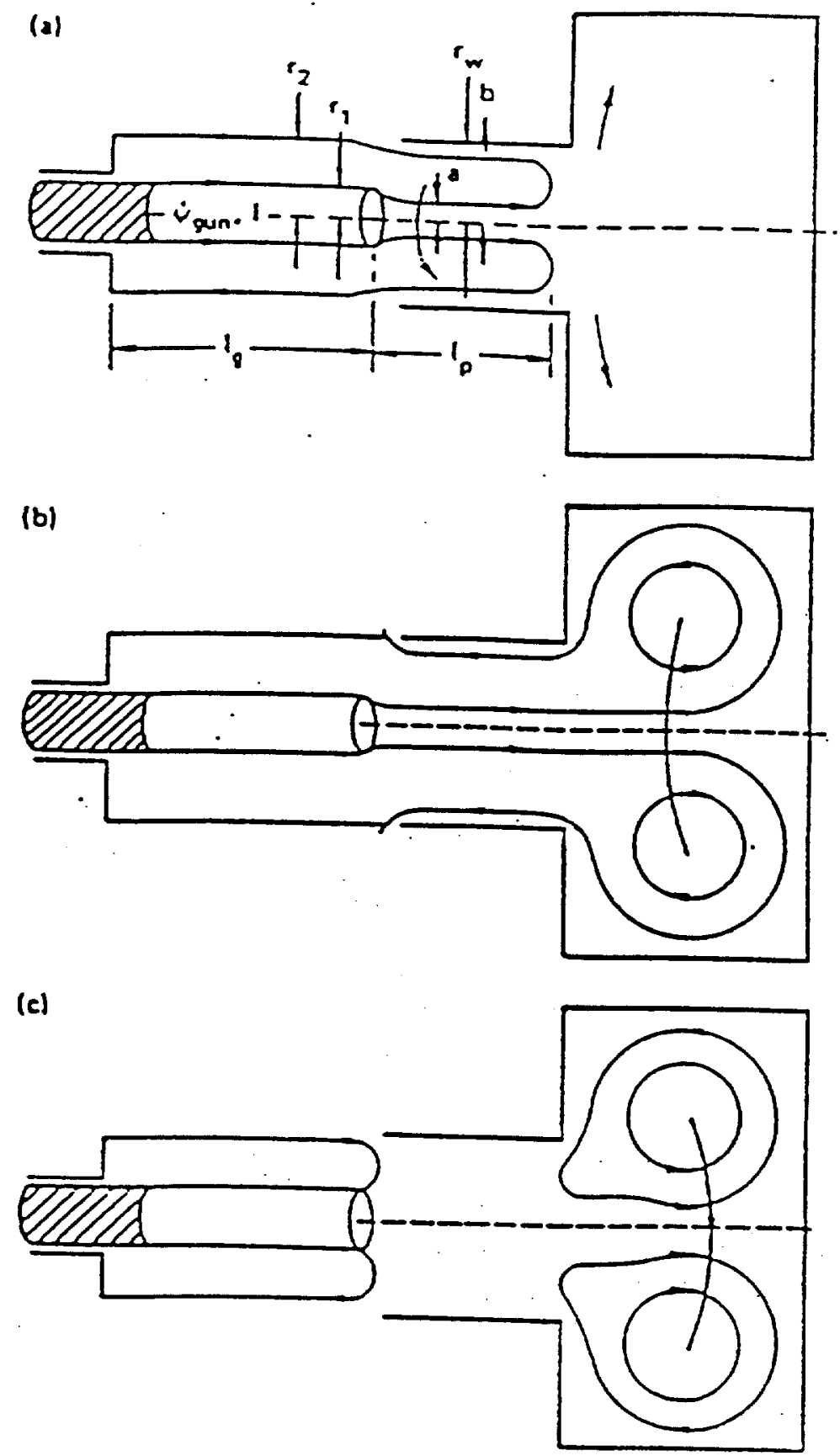

Fig. 2. Schematic of the formation of a spheromak. (From Jarboe ${ }^{38}$ ). The current, I, in the gun links the flux $\Psi_{\text {gun }}$ and expands into the flux conserver. (a) elongation of the initial flux, (b) expansion into the flux conserver, and (c) relaxation. In the sustainment mode, the magnetic field structure is maintained by continuous injection of helicity from the gun. 
In these decaying plasmas the energy confinement was limited by losses in the cold edge plasma. ${ }^{39}$ The lack of neutral-particle control at the spheromak edge, coupled to the constraints in the spheromak MHD equilibrium evolution, led to the global loop voltage (and thus global confinement) being dominated by plasma currents driven along the outer flux surfaces where the resistivity was very high due to electron-neutral collisions. ${ }^{39}$ Most of the magnetic energy dissipation was probably lost by charge exchange, particle transport, and conduction along open-field lines in the edge. Indeed, until the last series of experiments, the edge included a significant volume of open magnetic field lines, so that these losses were very large. In experiments on the CTX large flux conserver $(0.61 \mathrm{~cm})$, the global time was improved by careful magnetic design. These solid flux conserver experiments were funded not to study confinement, however, but only to achieve high temperature, low resistivity, and relatively long magnetic lifetimes suitable for explosive compression. ${ }^{40}$ As a consequence, the confinement during sustainment was not determined.

Further details and references can be found in several review articles. ${ }^{30,41,42,43}$

The critical information missing from previous experiments is the energy confinement time (and transport) in the core of the plasma and in sustained plasmas. Fowler developed a model ${ }^{1,8}$ based on the Rechester-Rosenbluth thermal conductivity applied to a plasma in which tearing modes carry helicity into the spheromak core. Extrapolation to reactors is very promising in that it predicts good confinement at the low level of turbulence needed to transport helicity at high electron temperatures. The model also predicts that ohmic ignition of a reactor is possible.

Experimental evidence for this hypothesis was examined in considerable detail by Hooper, et al., ${ }^{2}$ who concluded that it was well supported by experiments in decaying plasmas but that there is insufficient data from spheromaks sustained by helicity injection. They include discussion of other physics issues and of an experiment proposed to evaluate the energy confinement and the transition from an equilibrium supported by a flux conserver to one supported by external coils.

\subsection{Reactor opportunities ${ }^{*}$}

The route to improved reactors based on magnetic fusion energy may lie in the return to conceptually simple physics configurations rather than in refined engineering concepts for the present approach. ${ }^{44}, 45$ This will offer the potential to obtain the step-change in improvement needed for a truly viable product. Accordingly, of all the magnetic geometries explored to date, the

\footnotetext{
*Based in part on material from L. John Perkins
} 
spheromak arguably offers the best reactor potential: ${ }^{9}$ because of its simple geometry, no materials (first wall, vacuum vessel, blankets or magnets) link the torus. This leads to the following distinct advantages:

- Compact fusion power core with high mass power density: $\Rightarrow$ Potential for smaller unit size plants with good economics.

- Low complexity reactor geometry (no toroidal field coils, low technology normal-conducting equilibrium field coils, simple "pool" blankets): $\Rightarrow$ Increased reliability and maintainability; good availability prospects.

- Single, one-piece fusion power core: $\Rightarrow$ Factory fabrication and testing; simplified assembly; one-piece maintenance; one-piece disposal.

- Significantly cheaper and shorter development path for the series: Ignition $\rightarrow$ Engineering Test Reactor $\rightarrow$ DEMO $\rightarrow$ Commercial Prototype.

- External divertor system effected by the open geometry.

- Plasma sustained in steady-state by helicity injection: $\Rightarrow$ Greater inherent efficiency relative to conventional, non-inductive current-drive methods.

- Desirable core size and power outputs for other non-electric applications: Desalination, fission waste transmutation, fission/fusion hybrids, etc.

An artist's conception of a spheromak configuration appropriate for a reactor is shown in Fig. 3. Previous studies of the spheromak reactor, e.g. by Krakowski and coworkers at LANL, ' have demonstrated relatively low cost of electricity in a simple system which could be easily maintained. Modern reactor analysis techniques suggest that further improvements may be possible. Fowler, et al. ${ }^{7}$ estimate that ohmic ignition may be possible, eliminating the need for auxiliary heating; the result is based on application of energy confinement scaling to the geometry. ${ }^{46}$ Consequently, if the energy confinement and beta limit in a spheromak reactor can be demonstrated to be sufficiently good, this configuration may yield the best possible reactor available to harness magnetic fusion energy.

Analysis of this configuration ${ }^{47,48}$ indicates that it would make an excellent reactor if the energy confinement and other issues can be successfully worked out consistent with the drive of helicity from the plasma edge. 
Figure 3. Advanced spheromak reactor (artist's conception).

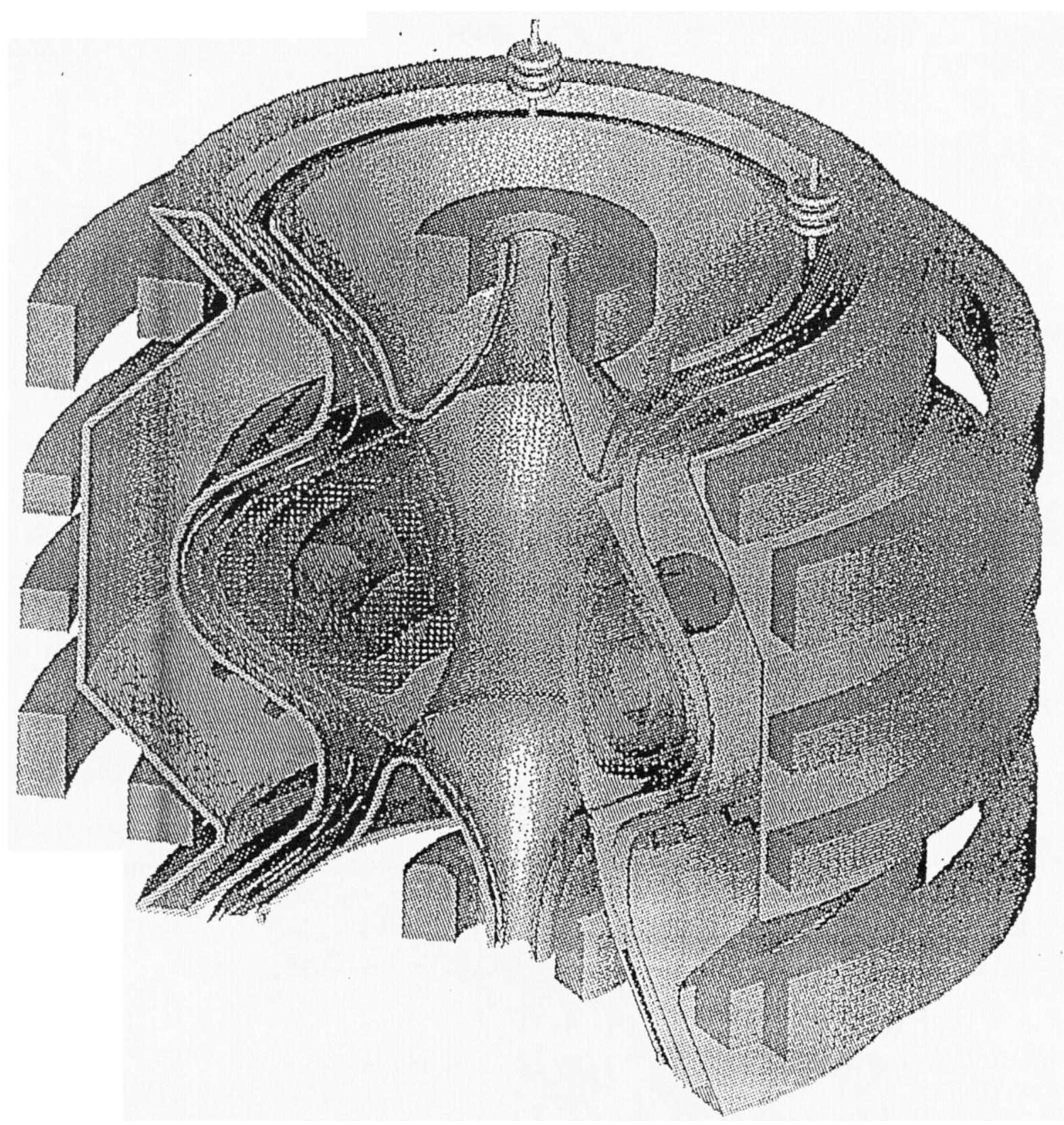




\section{References}

1. T. K. Fowler, Fusion Techn. 29, 206 (1996).

2. E. B. Hooper, J. H. Hammer, C. W. Barnes, J. C. Fernández, and F. J. Wysocki, "A re-examination of spheromak experiments and opportunities," Fusion Techn. 29, 191 (1996).

3. T. G. Cowling, Magnetohydrodynamics, (Interscience, NY, 1957).

4. T. R. Jarboe, F. J. Wysocki, J. C. Fernández, I. Henins, and G. J. Marklin, "Progress with Energy Confinement Time in the CTX Spheromak," Phys Fluids B, 2 1342, 1990.

5. L. A. Artsimovich, et al., "Experiments in tokamak devices," in Plasma Physics and Controlled Nuclear Fusion Research 1968, (IAEA, Vienna, 1969), Vol. I, page 157.

6. T. K. Fowler, "A Spheromak Ignition Experiment Reusing Mirror Fusion Test Facility (MFTF) Equipment," UCRL-ID-114696, September 28, 1993.

7. T. K. Fowler, J. S. Hardwick, and T. R. Jarboe, "On the Possibility of Ohmic Ignition in a Spheromak," Comments on Plasma Phys. Controlled Fusion 16, 91 (1994).

8. T. K. Fowler, "Heat Loss by Helicity Injection in Spheromaks," UCRL-ID116975, March 17, 1994.

9. R. L. Hagenson and R. A. Krakowski, "Steady-state spheromak reactor studies," Fusion Techn. 8, 1606 (1985); "The spheromak as a compact fusion reactor," LA-10908, March 1987.

10. M. N. Rosenbluth and M. N. Bussac, "MHD Stability of Spheromak," Nucl. Fusion 19, 489 (1979).

11. M. Yamada, et al., "Quasistatic formation of the spheromak plasma configuration," Phys. Rev. Lett. 46, 188 (1981).

12. G. C. Goldenbaum, J. H. Irby, Y. P. Chong, and G. W. Hart, "Formation of a spheromak plasma configuration," Phys. Rev. Lett. 44, 393 (1980).

13. W. C. Turner, E. H. A. Granneman, C. W. Hartman, D. S. Prono, J. Taska, and A. C. Smith Jr., "Production of Field-reversed Plasma with a Magnetized Coaxial Plasma Gun," J. Appl. Phys. 52, 175, 1981.

14. K. Watanabe, K. Ikegami, A. Ozaki, N. Satomi, and T. Uyama, "Compact toroidal plasma with toroidal and poloidal magnetic fields," J. Phys. Soc. Japan 50, 1823 (1981).

15. K. Kawai, Z. A. Pietrzyk, and H. T. Hunter, "Generation of poloidally rotating spheromaks by the conical theta pinch," Phys. Fluids 30, 2561 (1987). 
16. T. R. Jarboe, C. W. Barnes, D. A. Platts, and B. L. Wright, "A kinked Zpinch as the helicity source for spheromak generation and sustainment," Comments Plasma Phys. Controlled Fusion 9, 161 (1985).

17. W. C. Turner, G. C. Goldenbaum, E. H. A. Granneman, J. H. Hammer, C. W. Hartman, D. S. Prono, and J. Taska, "Investigations of the Magnetic Structure and the Decay of a Plasma-gun-generated Compact Torus," Phys. Fluids, 26, 1965 (1983).

18. T. R. Jarboe, et al., "Motion of a compact toroid inside a cylindrical flux conserver," Phys. Rev. Lett. 45, 1264 (1980).

19. C. Munson, A. Janos, F. Wysocki, and M. Yamada, "Experimental control of the spheromak tilting instability," Phys. Fluids 28, 1525 (1985).

20. F. J. Wysocki, "Experimental investigation of line tying effects on the spheromak tilt mode," Phys. Fluids 30, 482 (1987).

21. C. W. Barnes, T. R. Jarboe, G. J. Marklin, S. O. Knox, and I. Henins, "The impedance and energy efficiency of a coaxial magnetized plasma source used for spheromak formation and sustainment," Phys. Fluids B 2, 1871 (1990).

22. J. B. Taylor, "Relaxation of toroidal plasma and generation of reverse magnetic field," Phys. Rev. Lett. 33, 1139 (1974).

23. J. B. Taylor, "Relaxation and magnetic reconnection in plasmas," Rev. Mod. Phys. 58, 741 (1986).

24. L. Woltjer, "A theorem on force-free magnetic fields," Proc. Nat. Acad. Sci. USA 44, 489 (1958).

25. D. R. Wells and J. Norwood, Jr., "A variational approach to the dynamic stability of high-density plasmas in magnetic field containment devices," J. Plasma Phys. 3, 21 (1969).

26. J. H. Hammer, "Theoretical Aspects of Magnetic Helicity," In Advances in Compact Torus Research, page 75. (IAEA, Vienna, 1986). IAEA-TECDOC369 (Proceedings of a Technical Committee Meeting held in Sydney, Australia, 4-7 March 1985).

27. C. W. Barnes, et al., "Experimental determination of the conservation of magnetic helicity from the balance between source and spheromak," Phys. Fluids 29, 3415 (1986).

28. T. R. Jarboe, I. Henins, A. R. Sherwood, C. W. Barnes, and H. W. Hoida, "Slow formation and sustainment of spheromaks by a coaxial magnetized plasma source," Phys. Rev. Lett. 51, 39 (1983).

29. T. Uyama, et al., "Temporal evolution of the decaying spheromak in the CTCC-1 experiment," Nucl. Fusion 27, 799 (1987).

30. S. Shimamura, "Formation and sustainment of spheromak by using oscillating gun current with DC component," J. Phys. Soc. Japan 60, 4384 (1991).

31. B. R. Wright, et al., "Helicity conservation and energy confinement in CTX spheromaks," in Plasma Physics and Controlled Nuclear Fusion Research 1986, (IAEA, Vienna, 1987), Vol. II, page 519.

32. F. J. Wysocki, J. C. Fernández, T. R. Jarboe, and G. J. Marklin, "Evidence for 
a pressure-driven instability in the CTX spheromak," Phys. Rev. Lett. 61, 2457 (1988).

33. R. M. Mayo and G. J. Marklin, "Numerical calculation of Mercier beta limits in spheromaks," Phys. Fluids 31, 1812 (1988).

34. S. C. Jardin, "Ideal Magnetohydrodynamic stability of the spheromak configuration," Nucl. Fusion 22, 629 (1982).

35. E. B. Hooper, High-beta, Mercier-stable Spheromaks, Jan. 16, 1996 (unpublished).

36. J. H. Hammer, "MHD Tilting Modes for Nearly Spherical Compact Toroids with Arbitrary Plasma Pressure," Nucl. Fusion, 21, 488, 1981.

37. R. M. Mayo, D. J. Hurlburt, and J. C. Fernández, "Ion temperature profile deconvolution and corrections to confinement parameters in spheromaks," Phys. Fluids B 5, 4002 (1993).

38. T. R. Jarboe, "Review of Spheromak Research," Plasma Phys. Control. Fusion 36, 945 (1994).

39. J. C. Fernández, et al., "Energy confinement studies in spheromaks with mesh flux conservers," Nucl. Fusion 28, 1555 (1988).

40. J. Fernández, "Spheromak capable of accelerating hypervelocity projectiles," Defense Science Update 2, 1 (1990), LA-CP-90-395.

41. M. Yamada, Fusion Techn. 9, 38 (1986).

42. B. L. Wright, "Field reversed configurations and spheromaks," Nucl. Fusion 30, 1739 (1990).

43. J. C. Fernández, R. E. Chrien, F. J. Wysocki, R. M. Mayo, and I. Henins, "A view on advances in spheromak understanding and parameters," in Physics of Alternative Magnetic Confinement Schemes, S. Ortolani and E. Sindone (eds) (SiF, Bologna, 1991), page 191.

44. L. J. Perkins, J. H. Hammer, D. E. Baldwin, C. W. Hartman, “New Approaches to Fusion Energy," Bull. Amer. Phys. Soc, 38, 2053 (1993)

45. L. J. Perkins, J. H. Hammer, D. E. Baldwin, "Fusion, the Competition and the Need for Advanced Fusion Research," Seminar, DOE Office of Fusion Energy, Germantown, (Sept 1993)

46. T. K. Fowler and D. Hua, "Prospects for spheromak fusion reactors," J. Fusion Energy 14, 181 (1995).

47. E. B. Hooper and T. K. Fowler, "Spheromak Reactor: Physics Opportunities and Issues," Proc. 1996 American Nuclear Society Meeting, Reno, June 1620, 1996; LLNL Report UCRL-JC-122822.

48. T. K. Fowler and E. B. Hooper"Advanced Spheromak Reactor," Proc. 8th Intern. Conf. Emerging Nucl. Energy Systems, Obninsk, Russia, June 24-28, 1996; LLNL Report UCRL-JC-124363. 


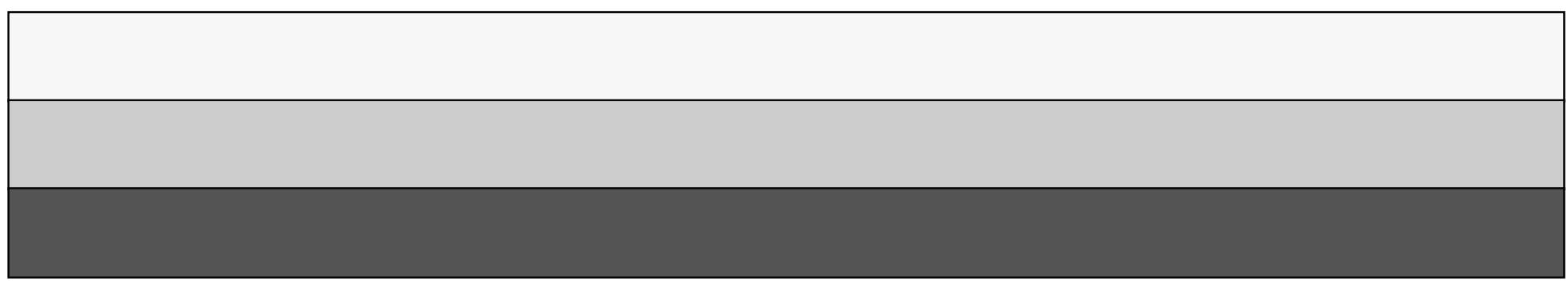

